\title{
Schur decomposition in the scaled boundary finite element method in elastostatics
}

\author{
M Li, H Song, H Guan and H Zhang \\ Griffith School of Engineering, Griffith University, Gold Coast, QLD, 4222, Australia \\ E-mail: m.li@griffith.edu.au
}

\begin{abstract}
The scaled boundary finite element method (SBFEM) was originally proposed for modelling elastodynamics in bounded and unbounded media. The method has demonstrated its superiority to the finite element method and the boundary element method when dealing with problems involving unbounded computational domains, or with difficulties of irregular frequencies and sharp corners. The SBFEM transforms the governing equations from partial differential equations to ordinary differential equations (ODEs). In addition, only the boundary of the study domain needs to be discretised which significantly reduces the computational cost. In the existing solution procedure, an eigenvalue problem of the Hamiltonian matrix, formulated from the coefficient matrices of ODEs, needs to be solved. Subsequently, the eigenvectors of the Hamiltonian matrix are arranged in a matrix form for the stiffness matrix in the nodal force-displacement relationship. However, the matrix formulated by the eigenvectors is close to singular when multiple eigenvalues with parallel eigenvectors exist, which leads to an inaccurate solution. In the present study, this problem is eliminated by using the Schur decomposition instead of the eigenvalue decomposition. A three-dimensional study of a cylindrical pile subjected to uniformly distributed load, is carried out. The performance and efficiency of the Schur decomposition are discussed in some detail for achieving more accurate solutions in using the SBFEM.
\end{abstract}

\section{Introduction}

The scaled boundary finite element method has been developed for the benefit of conveniently and efficiently handling problems with unbounded computational domains, or with irregular frequencies and sharp corners, where both the finite element method and the boundary element method find their limitations [1-3]. By transforming from conventional coordinate system to the scaled boundary coordinate system and using boundary discretisation with the scaled boundary finite elements, the governing equations formulating the physical problem are derived in the matrix form of ordinary differential equations with the dimensionless radial coordinate as a single variable. Subsequently, the nodal function is solved analytically from the scaled boundary finite element equation. In the existing solution procedure for elastostatics problems, a Hamiltonian matrix is formulated with the coefficient matrices of the scaled boundary finite element equation, whereby the nodal displacement function is the basic unknown function. A new intermediate function is introduced in relation to the nodal displacement function and the internal nodal load function. This leads to a reduction from a secondorder ordinary differential equation to a first-order differential equation at the expense of doubling the number of unknowns. The newly introduced intermediate function is hypothesised in the form of the power series, which leads to an eigenproblem of the Hamiltonian matrix in the solution procedure[4]. 
In real cases, however, not all the solutions of elastostatics problems are formulated by power functions only. Logarithmic terms do exist in the solution of certain problems with particular geometric configurations, material composition and boundary conditions [5-8]. In these cases, multiple eigenvalues might exist. These multiple eigenvalues may correspond to parallel eigenvectors indicating the existence of logarithmic terms in the solution which are not known in prior. Consequently, matrices formulated by the eigenvectors of the Hamiltonian matrix are rank-deficient and irreversible. In this case, the linear dependency of the eigenvectors results in the collapse of the eigenvalue decomposition solution of the scaled boundary finite element equation.

Deeks and Wolf $[9,10]$ investigated a two-dimensional unbounded domain problem governed by the Laplace equation using the SBFEM, in which case, the displacement amplitude is infinite in the near field. This infinite term is represented by introducing an additional logarithmic mode, associated with the rigid body translation, to the power series formulation of the solution. Song [11] proposed a matrix function solution in combination with the real Schur decomposition to address this multiple eigenvalues issue. Each term in the series is not restricted to being a power function. Unlike the work presented in $[9,10]$, Song's matrix function method does not require prior knowledge of the presence of the logarithmic terms. In addition, real Schur decomposition is more stable and suffers less from numerical difficulties than the eigenvalue decomposition.

In this paper, the real Schur decomposition is employed to deal with the underlying possible logarithmic terms which cannot be explored by the eigenvalue decomposition. By performing the Schur decomposition, the inverse of a close-to-singular matrix can be avoided by only inversing an upper triangular matrix. The Schur decomposition demonstrates its value as it allows the solution of the SBFEM to be more accurate.

Although more detailed descriptions can be found elsewhere in [3, 12-15], fundamental SBFEM concepts and derivations are introduced briefly for the purpose of integrity. The solution procedure using the Schur decomposition will be presented subsequently. A three-dimensional cylindrical pile subjected to uniformly distributed load serves as a case study to demonstrate the efficiency of the proposed method.

\section{Scaled boundary finite element method with eigenvalue decomposition}

\subsection{Scaled boundary finite element equation}

The governing equations for three-dimensional elastostatics omitting body load expressed in displacement amplitudes $\{u\}=\{u(\hat{x}, \hat{y}, \hat{z})\}=\left[u_{x}, u_{y}, u_{z}\right]^{T}$ in Cartesian coordinates $\hat{x}, \hat{y}$ and $\hat{z}$ are shown in a vector form as:

$$
[L]^{T}\{\sigma\}=0
$$

where $[L]$ represents the differential operator. The stress amplitudes $\{\sigma\}$ follow from Hooke's law with the material matrix $[D]$ as:

$$
\{\sigma\}=[D]\{\varepsilon\}
$$

The strain amplitudes $\{\varepsilon\}$ and displacement amplitudes are related by $[L]$ in the form of :

$$
\{\varepsilon\}=[L]\{u\}
$$

The differential operator $[L]$ is formulated in the scaled boundary coordinate as:

$$
[L]=\left[b^{1}(\eta, \zeta)\right] \frac{\partial}{\partial \xi}+\frac{1}{\xi}\left(\left[b^{2}(\eta, \zeta)\right] \frac{\partial}{\partial \eta}+\left[b^{3}(\eta, \zeta)\right] \frac{\partial}{\partial \zeta}\right)
$$

with $\left[b^{1}(\eta, \zeta)\right],\left[b^{2}(\eta, \zeta)\right]$ and $\left[b^{3}(\eta, \zeta)\right]$ being determined on the boundary discretisation and independent of the radial coordinate $\xi$. 
The point on the structure surface is denoted as $(x, y, z)$ as shown in figure 1 , which can be formulated as:

$$
\begin{aligned}
& x(\eta, \zeta)=[N(\eta, \zeta)]\{x\} \\
& y(\eta, \zeta)=[N(\eta, \zeta)]\{y\} \\
& z(\eta, \zeta)=[N(\eta, \zeta)]\{z\}
\end{aligned}
$$

in terms of the discretised nodal coordinate on the boundary $(\{x\},\{y\},\{z\})$ and the mapping functions $[N(\eta, \zeta)]$.

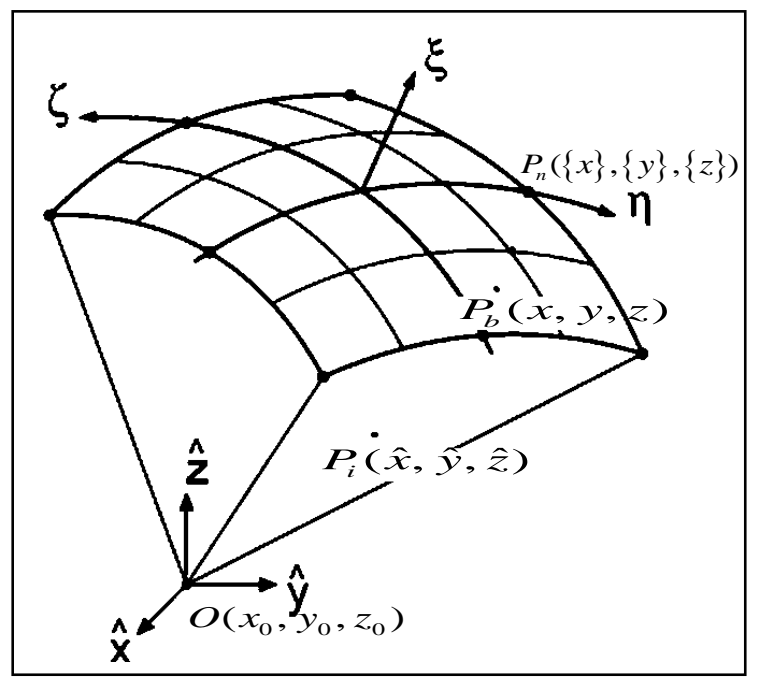

Figure 1. Points definition in the scaled boundary coordinate system.

Using the same shape function $[N(\eta, \zeta)]$ as for the boundary, the displacement is expressed in the form of:

$$
\{u(\xi, \eta, \zeta)\}=[N(\eta, \zeta)]\{u(\xi)\}
$$

where $\{u(\xi)\}$ is the nodal displacement function.

From (2) - (4), the stress and strain amplitudes are expressed as:

$$
\begin{gathered}
\{\varepsilon\}=\{\varepsilon(\xi, \eta, \zeta)\}=\left[B^{1}\right]\{u(\xi)\}_{, \xi}+\frac{1}{\xi}\left[B^{2}\right]\{u(\xi)\} \\
\{\sigma\}=\{\sigma(\xi, \eta, \zeta)\}=[D]\left(\left[B^{1}\right]\{u(\xi)\}_{, \xi}+\frac{1}{\xi}\left[B^{2}\right]\{u(\xi)\}\right)
\end{gathered}
$$

with two matrices $\left[B^{1}(\eta, \zeta)\right]$ and $\left[B^{2}(\eta, \zeta)\right]$ being formulated as:

$$
\begin{gathered}
{\left[B^{1}(\eta, \zeta)\right]=\left[b^{1}(\eta, \zeta)\right][N(\eta, \zeta)]} \\
{\left[B^{2}(\eta, \zeta)\right]=\left[b^{2}(\eta, \zeta)\right][N(\eta, \zeta)]_{, \eta}+\left[b^{3}(\eta, \zeta)\right][N(\eta, \zeta)]_{, \zeta}}
\end{gathered}
$$

Through a series of manipulations, the governing differential equation (1) is transformed into the matrix-form second order ordinary differential equation (11) with respect to displacement

$$
\left[E^{0}\right] \xi^{2}\{u(\xi)\}_{, \xi \xi}+\left(2\left[E^{0}\right]+\left[E^{1}\right]^{T}-\left[E^{1}\right]\right) \xi\{u(\xi)\}_{, \xi}+\left(\left[E^{1}\right]^{T}-\left[E^{2}\right]\right)\{u(\xi)\}=0
$$

where the coefficient matrices are defined as: 


$$
\begin{aligned}
& {\left[E^{0}\right]=\int_{-1}^{1} \int_{-1}^{1}\left[B^{1}(\eta, \zeta)\right]^{T}[D]\left[B^{1}(\eta, \zeta)\right]|J(\eta, \zeta)| d \eta d \zeta} \\
& {\left[E^{1}\right]=\int_{-1}^{1} \int_{-1}^{1}\left[B^{2}(\eta, \zeta)\right]^{T}[D]\left[B^{1}(\eta, \zeta)\right]|J(\eta, \zeta)| d \eta d \zeta} \\
& {\left[E^{2}\right]=\int_{-1}^{1} \int_{-1}^{1}\left[B^{2}(\eta, \zeta)\right]^{T}[D]\left[B^{2}(\eta, \zeta)\right]|J(\eta, \zeta)| d \eta d \zeta}
\end{aligned}
$$

\subsection{Eigenvalue decomposition solution procedure and its shortcomings}

Formulating the nodal displacement function $\{u(\xi)\}$ and the internal nodal force function $\{Q(\xi)\}$ in the following manner leading to an intermediate variable $\{X(\xi)\}$ with twice the number of unknowns as:

$$
\{X(\xi)\}=\left\{\begin{array}{c}
\xi^{0.5}\{u(\xi)\} \\
\xi^{-0.5}\{Q(\xi)\}
\end{array}\right\} .
$$

Equation (11) is transformed into a series of first-order ordinary differential equations, which can be expressed in matrix form as:

$$
\xi\{X(\xi)\}_{, \xi}=-[Z]\{X(\xi)\}
$$

with the Hamiltonian matrix $[Z]$ formulated using the coefficient matrices of the scaled boundary finite element equation as:

$$
[Z]=\left[\begin{array}{cc}
{\left[E^{0}\right]^{-1}\left[E^{1}\right]^{T}-0.5[I]} & -\left[E^{0}\right]^{-1} \\
-\left[E^{2}\right]+\left[E^{1}\right]\left[E^{0}\right]^{-1}\left[E^{1}\right]^{T} & -\left[E^{1}\right]\left[E^{0}\right]^{-1}+0.5[I]
\end{array}\right]
$$

By inspection, the solution of (14) can be formulated using a finite power series as:

$$
\{X(\xi)\}=c_{1} \xi^{-\lambda_{1}}\left\{\phi_{1}\right\}+c_{2} \xi^{-\lambda_{2}}\left\{\phi_{2}\right\}+\cdots+c_{n} \xi^{-\lambda_{n}}\left\{\phi_{n}\right\}
$$

with $n$ denoting the dimension of the Hamiltonian matrix $[Z]$.

Substituting (15) into (14) leads to the eigenproblem:

$$
[Z]\left\{\phi_{i}\right\}=\lambda_{i}\left\{\phi_{i}\right\}
$$

where $\lambda_{i}$ is the eigenvalue of $[Z]$ and $\left\{\phi_{i}\right\}$ is the corresponding eigenvector.

Reformulate $\{X(\xi)\}$ in matrix form:

$$
\{X(\xi)\}=\left[\begin{array}{ll}
{\left[\Phi_{11}\right]} & {\left[\Phi_{12}\right]} \\
{\left[\Phi_{21}\right]} & {\left[\Phi_{22}\right]}
\end{array}\right]\left[\begin{array}{ll}
{\left[\xi^{\Lambda_{1}}\right]} & \\
& {\left[\xi^{\Lambda_{2}}\right]}
\end{array}\right]\left\{\begin{array}{l}
\left\{C_{1}\right\} \\
\left\{C_{2}\right\}
\end{array}\right\}
$$

Note, if $\lambda$ is the eigenvalue of $[Z]$, then $-\lambda, \bar{\lambda}$ (conjugate complex number) and $-\bar{\lambda}$ are eigenvalues of $[Z]$. The eigenvalues $\lambda_{i}$ of matrix $[Z]$ can be arranged in such a way that all the eigenvalues in $\Lambda_{1}$ have positive real parts, and all the eigenvalues in $\Lambda_{2}$ have negative real parts.

According to (17) and (13), $\{u(\xi)\}$ and $\{Q(\xi)\}$ can be expressed as:

$$
\begin{aligned}
& \{u(\xi)\}=\xi^{-0.5}\left(\left[\Phi_{11}\right]\left[\xi^{\Lambda_{1}}\right]\left\{C_{1}\right\}+\left[\Phi_{12}\right]\left[\xi^{\Lambda_{2}}\right]\left\{C_{2}\right\}\right) \\
& \{Q(\xi)\}=\xi^{+0.5}\left(\left[\Phi_{21}\right]\left[\xi^{\Lambda_{1}}\right]\left\{C_{1}\right\}+\left[\Phi_{22}\right]\left[\xi^{\Lambda_{2}}\right]\left\{C_{2}\right\}\right)
\end{aligned}
$$

where the integral constants $\left\{C_{1}\right\}$ and $\left\{C_{2}\right\}$ are to be determined based on the boundary conditions.

The displacement amplitude at the scaling centre where $\xi=0$ of the bounded domain should be finite. Since the real parts of $\lambda_{i}$ in $\Lambda_{2}$ are negative, (18) and (19) are reduced to 


$$
\{u(\xi)\}=\xi^{-0.5}\left[\Phi_{11}\right]\left[\xi^{\Lambda_{1}}\right]\left\{C_{1}\right\}
$$

and

$$
\{Q(\xi)\}=\xi^{+0.5}\left[\Phi_{21}\right]\left[\xi^{\Lambda_{1}}\right]\left\{C_{1}\right\}
$$

The constant vector $\left\{C_{1}\right\}$ is determined according to the condition at the boundary of the bounded domain where $\xi=1$.

On the other hand, formulate the nodal force-nodal displacement relationship on the boundary as:

$$
\{R\}=[K]\{u\}
$$

where $\{R\}$ is the nodal force vector, and $\{u\}$ is the nodal displacement vector. To calculate the staticstiffness matrix $[K]$ defined in (22), formulate (20) and (21) at $\xi=1$, with the internal nodal force on the boundary $\{Q(\xi=1)\}$ being balanced by the nodal force, and compare with (22) leads to the staticstiffness matrix:

$$
[K]=\left[\Phi_{21}\right]\left[\Phi_{11}\right]^{-1}
$$

After $\left\{C_{1}\right\}$ is determined, the nodal displacement function $\{u(\xi)\}$ along the line defined by connecting the scaling centre and the corresponding node on the boundary is analytically obtained from (20). Accordingly, the displacement amplitude at any point can be determined by interpolation using the expression $\{u(\xi, \eta, \zeta)\}=[N(\eta, \zeta)]\{u(\xi)\}$.

However, the eigenvalue method may not provide complete general solution. Inaccurate results may be incurred due to the rank-deficiency of the matrix $\left[\Phi_{11}\right]$. One of the possibilities of the rankdeficiency of the matrix maybe caused by the parallel eigenvectors corresponding to the multiple eigenvalues or near-multiple eigenvalues, which results from the logatithmic terms in the solution as discussed in [11]. Song [11] proposed a matrix function solution with real Schur decomposition to deal with the power functions, logarithmic functions and their transitions in the solution.

\section{Schur decomposition solution procedure}

The Schur decomposition of the Hamiltonian matrix $[Z]$ can be expressed as:

$$
[Z]=[V][S][V]^{T}
$$

where $[V]$ is an orthogonal matrix and $[S]$ is a block upper triangular matrix with 1-by-1 and 2-by-2 blocks on the diagonal. The eigenvalues are revealed by the diagonal elements and blocks of $[S]$, while the columns of $[\mathrm{V}]$ provide a basis with much better numerical properties than a set of eigenvectors [16].

Partitioning $[S]$ and $[V]$ into submatrices of equal size as:

$$
[S]=\left[\begin{array}{cc}
{\left[S_{n}\right]} & * \\
0 & {\left[S_{p}\right]}
\end{array}\right] \text {, and }[V]=\left[\begin{array}{cc}
{\left[V_{u 1}\right]} & {\left[V_{u 2}\right]} \\
{\left[V_{q 1}\right]} & {\left[V_{q 2}\right]}
\end{array}\right],
$$

where $*$ stands for a real matrix. The diagonal elements of matrix $\left[S_{n}\right]$ are negative and those of matrix $\left[S_{p}\right]$ are positive. Block-diagonalising $[S]$ using an upper-triangular matrix and using (24) leads to:

$$
[\Psi]^{-1}[Z][\Psi]=\left[\begin{array}{cc}
{\left[S_{n}\right]} & 0 \\
0 & {\left[S_{p}\right.}
\end{array}\right]
$$

Similar to the formulation in (17), the general solution of (14) is expressed as: 


$$
\{X(\xi)\}=\left[\begin{array}{ll}
{\left[\Psi_{u 1}\right]} & {\left[\Psi_{u 2}\right]} \\
{\left[\Psi_{q 1}\right]} & {\left[\Psi_{q 2}\right]}
\end{array}\right]\left[\begin{array}{cc}
\xi^{-\left[S_{n}\right]} & \\
& \xi^{-\left[S_{p}\right]}
\end{array}\right]\left\{\begin{array}{l}
\left\{C_{1}\right\} \\
\left\{C_{2}\right\}
\end{array}\right\}
$$

Therefore, $\{u(\xi)\}$ and $\{Q(\xi)\}$ can be expressed as:

$$
\begin{gathered}
\{u(\xi)\}=\xi^{-0.5}\left(\left[\Psi_{u 1}\right] \xi^{-\left[S_{n}\right]}\left\{C_{1}\right\}+\left[\Psi_{u 2}\right] \xi^{-\left[S_{n}\right]}\left\{C_{2}\right\}\right) \\
\{Q(\xi)\}=\xi^{+0.5}\left(\left[\Psi_{q 1}\right] \xi^{-\left[S_{n}\right]}\left\{C_{1}\right\}+\left[\Psi_{q 2}\right] \xi^{-\left[S_{p}\right]}\left\{C_{2}\right\}\right)
\end{gathered}
$$

The following solution procedure is the same as that from the eigenvalue decomposition solution procedure.

\section{Case study}

The deformation of a cylindrical pile subjected to uniformly distributed load is examined herein. The cylindrical pile with a radius of $a$ and a height of $H$ subjected to uniformly distributed pressure $p$ is shown in figure 2. The bottom of the cylinder pile is fixed. The pile is assumed to be a plain concrete pile with Young's modulus $E$ and Poisson's ratio $v$, and exhibit pure elastic property. The magnitudes of the dimensions and the properties of the pile are listed in table 1.
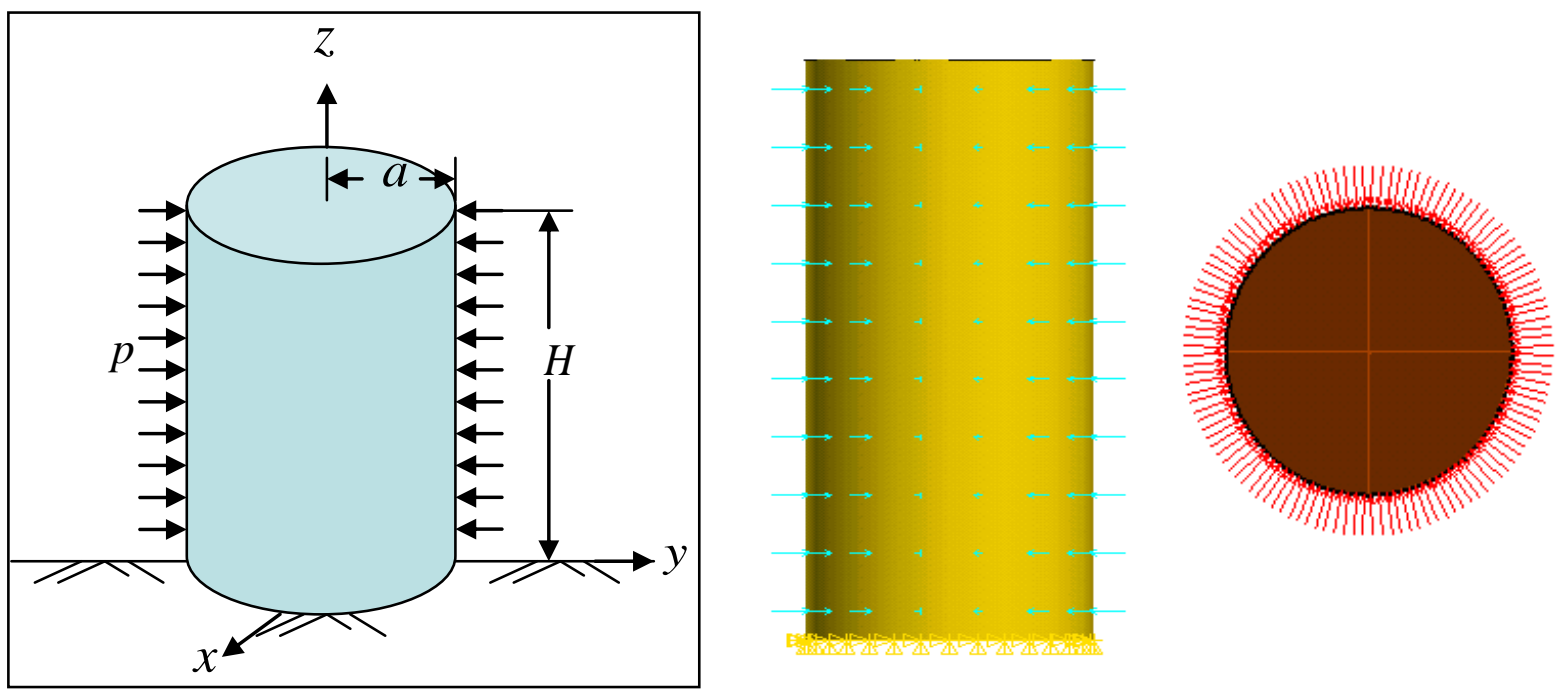

Figure 2. Cylindrical pile subjected to uniformly distributed load.

Table 1. Parameters for the cylinder pile

\begin{tabular}{cccccc}
\hline Parameters & $H(\mathrm{~m})$ & $a(\mathrm{~m})$ & $E(\mathrm{~Pa})$ & $v$ & $p(\mathrm{~Pa})$ \\
\hline Magnitude & 10 & 1 & $2.8 \times 10^{10}$ & 0.25 & $3 \times 10^{8}$ \\
\hline
\end{tabular}

The scaling center is chosen at the bottom center of the pile. The circumferential boundary, as well as the top surface of the cylinder is discretised with quadratic eight-node quadrilateral isoparametric elements with the shape functions listed in (27).

Schur decomposition solution procedure is employed. The convergence test shows that 8 elements are needed for the circumferential direction discretisation, 1 element for the radius direction and 16 elements for the height of the pile. The displacement at the top of the pile in the $x$ direction converges to $8.03571 \mathrm{~mm}$, and that in the $z$ direction is $48.82111 \mathrm{~mm}$. An example of the discretisation scheme is illustrated in figure 3.

The FEM analysis for the same problem is carried out for comparison. Three dimensional 20-node hexahedral solid elements are used in the FEM model, as shown in figure 4. A convergence test shows 
that 28 elements are needed in the circumferential direction, 5 elements in the radius direction and 50 elements along the height of the pile. The displacement at the top of the pile in the $x$ direction converges to $8.03571 \mathrm{~mm}$, and that in the $z$ direction reaches $52.42417 \mathrm{~mm}$.

$$
\begin{array}{ll}
N_{1}(\eta, \zeta)=-\frac{1}{4}(\eta-1)(\zeta-1)(\eta+\zeta+1) & N_{2}(\eta, \zeta)=\frac{1}{2}\left(\eta^{2}-1\right)(\zeta-1) \\
N_{3}(\eta, \zeta)=\frac{1}{4}(\zeta-1)\left(-\eta^{2}+\zeta \eta+\zeta+1\right) & N_{4}(\eta, \zeta)=-\frac{1}{2}(\eta+1)\left(\zeta^{2}-1\right) \\
N_{5}(\eta, \zeta)=\frac{1}{4}(\eta+1)(\zeta+1)(\eta+\zeta-1) & N_{6}(\eta, \zeta)=-\frac{1}{2}\left(\eta^{2}-1\right)(\zeta+1) \\
N_{7}(\eta, \zeta)=\frac{1}{4}(\eta-1)(\zeta+1)(\eta-\zeta+1) & N_{8}(\eta, \zeta)=\frac{1}{2}(\eta-1)\left(\zeta^{2}-1\right)
\end{array}
$$

The displacement distributions from both SBFEM and FEM models are plotted in figure 5. The solid line, the dashed line and the broken line represent the displacement from FEM results. Those dots, pentagons and crosses are from the SBFEM results. The discrepancy of the displacement in the $z$ direction along the height of the pile between these two models requires further error investigation, but the comparison shows the potential of SBFEM using the Schur decomposition.

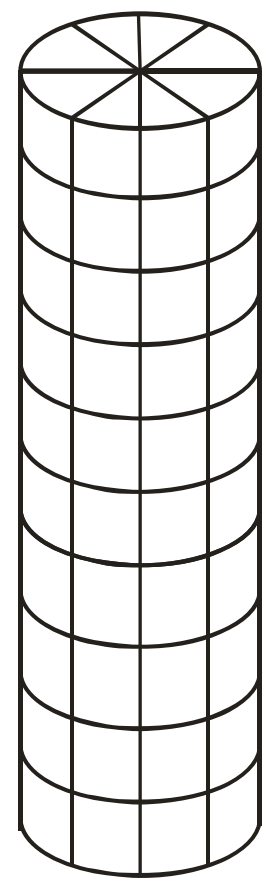

Figure 3. SBFEM discretisation of the pile

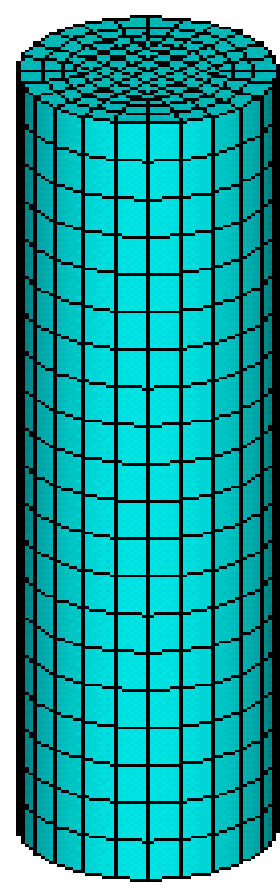

Figure 4. FEM discretisation of the pile

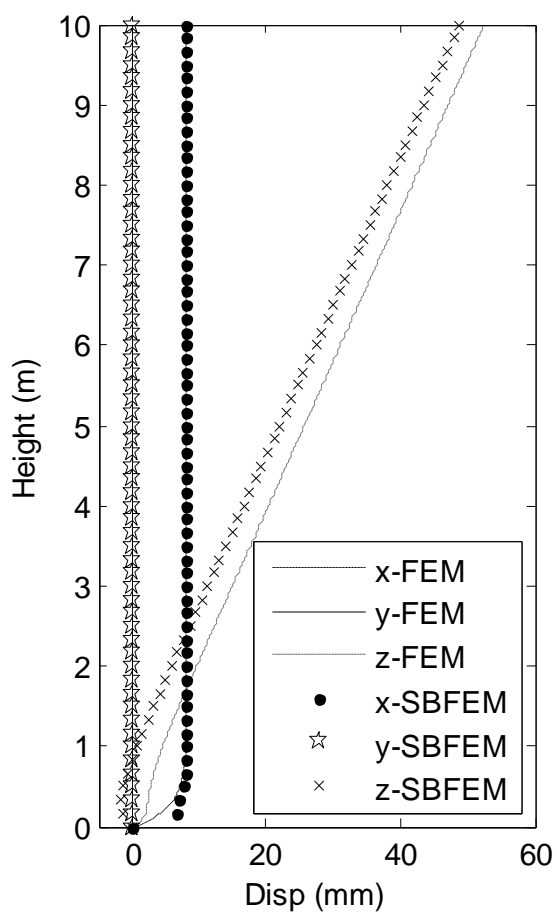

Figure 5. Displacement comparison of the pile from SBFEM and FEM models

In order to show the superiority of the Schur decomposition over the eigenvalue decomposition in the scaled boundary finite element equation solution procedure, a comparison of the results from these two methods is performed. The radius displacement and vertical displacement of any point on the edge of the top surface of the pile are plotted versus different discretisation scheme in figure 6 . The numbers along the abscissa denotes the discretisation scheme, for example, $6 \times 10 \times 1$ explains the numbers of elements in the circumferential direction, vertical direction and radial direction of the pile are 6,10 and 1 respectively. It is found that by using Schur decomposition, no prior knowledge of the potential multiple eigenvalues is required and no complex number operation is needed as the case in eigenvalue decomposition. The parallel-eigenvector issue can be successfully avoided. Schur 
decomposition tends to give more stable and accurate results compared with eigenvalue decomposition, as shown in figure 6. Furthermore, the eigenvalue decomposition consumes more computational memory. With same computer configuration, schur decomposition allows finer discretisation, whereas eigenvalue decomposition prohibits more accurate results. In this case study, the eigenvalue decomposition is suspended when the discretisation scheme is $8 \times 16 \times 1$.
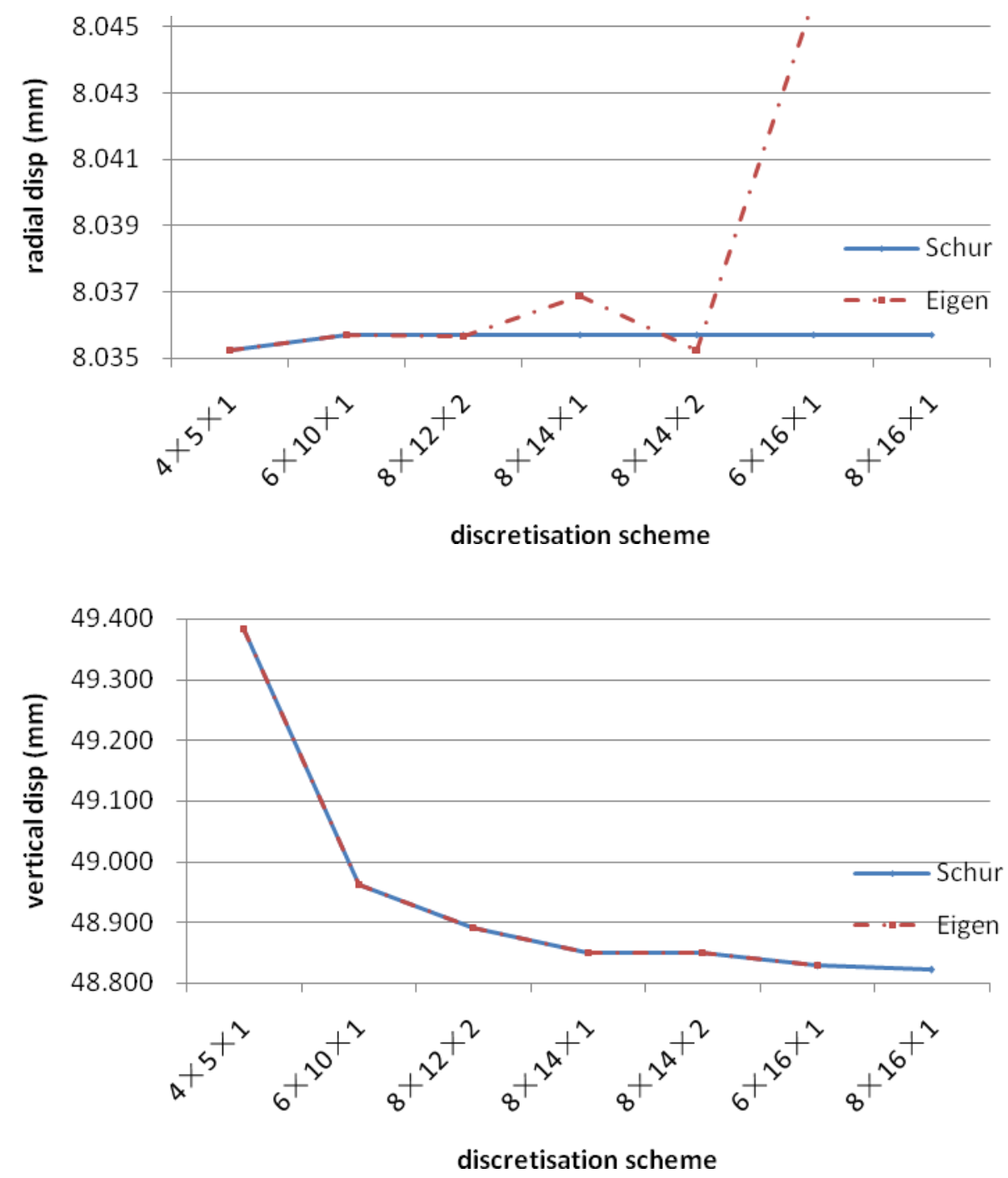

Figure 6. Displacement of an arbitrary point on the edge of the top surface of the pile using Schur decomposition and eigenvalue decomposition

\section{Conclusions}

The SBFEM has been developed for more than a decade and has demonstrated its broad application in various fields such as structural engineering, costal engineering and geotechnical engineering. It outperforms other numerical methods by combining all the following advantages in itself: (1) reducing the governing equation from the partial differential equation to ordinary differential equation; (2) reducing the spatial dimension of the problem by one and therefore the data preparation and computational effort by discretising the boundary only; (3) not requiring fundamental solution as is the 
case with boundary element method; (4) easily solving problems with boundary conditions at infinity. The existing solution procedure of the scaled boundary finite element equation involves an eigenvalue decomposition of a Hamiltonian matrix. The underlying multiple eigenvalues, corresponding to the potential logarithmic terms, which might not be known beforehand, reveal the numerical difficulties in the eigenvalue decomposition. Instead, Schur decomposition is a valid alternative since it eliminates this problem and provides a more stable and accurate solution irrespective of the existence of the logarithmic terms. Furthermore, no manipulations of complex numbers as in the eigenvalue decomposition are required. In this paper, Schur decomposition is used with SBFEM in a static analysis of a cylindrical pile subjected to uniformly distributed load. The results show that the present procedure works fairly well solving problems without the prior knowledge of the potential existence of the multiple eigenvalues and provides more stable and accurate results than the eigenvalue decomposition method.

\section{References}

[1] Song C M and Wolf J P 1997 Comput. Methods Appl. Mech. Engrg. 147 329-55

[2] Song C M and Wolf J P 1998 Comput. Methods Appl. Mech. Engrg. 164 249-64

[3] Wolf J P and Song C M 2001 Comput. Methods Appl. Mech. Engrg. 190 5551-68

[4] Wolf J P 2003 The Scaled Boundary Finite Element Method

[5] Sinclair G B 2000 J. Appl. Mech. ASME 67 219-23

[6] Gadi K S, et al. 2000 Eng. Fract. Mech. 65 645-64

[7] Chen D H 1996 Int. J. Fract. 75 357-78

[8] Sinclair G B $1999 \mathrm{~J}$. Appl. Mech. ASME 66 556-60

[9] Deeks A J and Wolf J P 2002 Int. J. Numer. Anal. Methods Geomech. 26 1031-57

[10] Deeks A J and Wolf J P 2003 Comput. Struct. 81 1525-37

[11] Song C M 2004 Comput. Methods Appl. Mech. Engrg. 193 2325-56

[12] Song C M and Wolf J P 2000 Comput. Struct. 78 211-25

[13] Wolf J P and Song C M 2000 Comput. Struct. 78 191-210

[14] Song C M and Wolf J P 1999 Comput. Methods Appl. Mech. Engrg. 180 117-35

[15] Deeks A J and Wolf J P 2002 Comput. Mech. 28 489-504

[16] Paige C and Van Loan C 1981 Linear Algebra Appl. 41 11-32 